\title{
EDITORIAL
}

\section{Introducing Modern Italy's new editors}

As the new editors of Modern Italy for the period 2015-2019, we are very conscious of the high bar set by our predecessors, Philip Cooke and John Foot. Together they guided the transition to ScholarOne's manuscript flow system, increased the journal's already dynamic range of publication, and further enhanced its international reputation among scholars of Italy as well as the academic publishing industry. They achieved all this during what their 2010 editorial rightly described as a 'critical time for Italian studies in the UK'. We thank them and their editorial team warmly for handing over to us, and to ASMI, a journal in excellent form, despite the very real challenges of the times.

Five years on, the context for research and teaching in Italian studies seems no less critical. The situation for humanities scholars worldwide is challenging, as teaching and learning priorities shift, and fiscal pressures are increasingly felt by scholars whose work does not always readily match templates of rational utility, social impact, or direct market relevance. We believe, though, that in this context journals such as Modern Italy, which provide authoritative platforms for rigorous research that both showcases and stimulates deep reflection on human societies and cultures across time and space, are all the more valuable.

One of the journal's particular strengths - like the Association that created it - lies in its role as a meeting point between disciplines, and in the forum it offers to research on Italy covering a myriad of angles and theoretical approaches. As editors we will continue to foster this breadth and depth. Indeed, we believe that Italy, laden with the sorts of social, political, and economic complexities to which humanities scholars are particularly drawn, can provide a special focal point for the analysis of questions whose significance is far from unique to Italy. For this reason, in addition to welcoming close-grained studies of Italy on its own terms, we would also like to encourage the submission of comparative and transnational research that situates Italy, whether historical or contemporary, in a broader context, vis-à-vis both Europe and the wider world.

For the first time since its inception nearly 20 years ago the journal will be edited by an international team. We look forward to developing the journal's global reputation, to building on relationships with reviewers and contributors established by previous editors, and to creating new dialogues, with, for example, specialists working on other European countries and beyond. We are very aware that editing a journal presents challenges that are best met through close collaboration, so we are delighted to have the opportunity to work closely with our associate editors, Andrea Hajek and Giacomo Lichtner, and bookreview editors Nick Carter and (from August 2015) Rebecca Clifford. 
We will continue to rely gratefully on the generosity of the journal's ever widening range of expert reviewers, and we look forward to engaging with the knowledge and views of the distinguished group of scholars who form the editorial board as we guide Modern Italy over the next five years.

Penelope Morris University of Glasgow

Mark Seymour University of Otago 